\title{
Placebo-Driven Clinical Trials of Transfer Point Glucan \#300 in Children with Chronic Respiratory Problems: III. Clinical Findings
}

\author{
${ }^{1}$ Vetvicka Vaclav, ${ }^{2}$ Richter Josef, ${ }^{3}$ Svozil Vladimir, \\ ${ }^{2}$ Rajnohova Dobiasova Lucie and ${ }^{2}$ Kral Vlastimil \\ ${ }^{1}$ Department of Pathology, University of Louisville, Louisville, KY, USA \\ ${ }^{2}$ Regional Institute of Public Health, Ústí nad Labem, Czech Republic, Europe \\ ${ }^{3}$ Sanatorium Edel, Zlaté Hory, Czech Republic, Europe
}

Received 2013-06-28, Revised 2013-07-31; Accepted 2013-08-01

\begin{abstract}
The role of glucan in stimulation of immune reactions has been studied for decades. In this report we focused on the effects of orally administered glucan in children with chronic respiratory problems. We measured the physical endurance using a 6MWT test and the levels of eNO in 50 children aged 8-12 years and evaluated the effects of $100 \mathrm{mg} / \mathrm{d}$ oral dose of glucan. We found significant improvements in physical indurance and exhaled nitric oxide in glucan-treated children. In addition, strong improvements in general conditions were found. Short-term oral application of natural immunomodulator $\beta$-glucan enhancers the overall health and regulation of energetic metabolism in children with chronic respiratory problems.
\end{abstract}

Keywords: Glucan, Children, Physical Endurance, eNO, Placebo

\section{INTRODUCTION}

$\beta$-glucans belong to a group of biologically active natural compounds called biological response modifiers. These substances represent highly conserved structural components of cell walls in fungi, yeast, grain and seaweed. Generally, $\beta$-glucan is a chemical name of a polymer of $\beta$-glucose. Usually exists as a homopolymer of glucose having a linear molecule with (1-3)- $\beta$-Dglycosilic linkages or a branched one with side chains bound by (1-6)- $\beta-D-$-glycosilic linkages. Since 1983, several forms of mushroom-derived $\beta$-glucan have been approved in Japan for treatment of cancer patients (Hamano et al., 1999). In other countries, glucan is still only a supplement. However, more and more studies are now focused on the use of glucan in clinical practice, including numerous clinical trials (Bergendiova et al., 2011; Lehne et al., 2005; Auinger et al., 2013; Jesenak et al., 2013a).

Extensive research in this field yielded new types of glucan with higher purity and effectiveness that are able to significantly stimulate defense mechanisms not only against microbes but also toxic and other influences (Vetvicka and Vetvickova, 2008; 2013). In addition, strong effects on hypertension, cholesterol levels, arthritis and blood sugar regulation were observed (Vetvicka and Vetvickova, 2007).

Glucan acts via binding to specific receptors such as CR3 or Dectin-1 and via intracellular signaling (Dennehy et al., 2008) thus influencing numerous cell types including monocytes, neutrophils, dendritic cell and NK cells. In addition, glucan manifested significant effects in prevention or modulation of infectious diseases (Renzetti et al., 2009; Ramberg et al., 2010; Novak and Vetvicka, 2008). Some recent observations showed that glucan has palative anti-allergic effects ( $\mathrm{Ku}$ et al., 2012), even in children with respiratory problems (Jesenak et al., 2013b). The possibility that these effects might be manifested via decreasing proinflammatory cytokines such as IL- 6 and TNF- $\alpha$ and increasing of secretion of IL-10 and accession of cellular antioxidants was suggested (Senoglu et al., 2008).

Corresponding Author: Vetvicka Vaclav, Department of Pathology, University of Louisville, Louisville, KY, USA 
These findings led us to our clinical study on the effects of short-term administration of glucan on health parameters of children with chronic respiratory problems, allergies and asthma.

Our study was directed toward the evaluation of the effects on some parameters of mucosal immunity, testing for possible side effects and effects on important parameters of physical performance and improvements of quality of lung functions damaged by contaminated environment.

\section{MATERIALS AND METHODS}

\subsection{Protocol}

The same protocol that was previously described (Vetvicka et al., 2013) was used throughout this study. Briefly, a randomized, double-blind, placebo-controlled trial compared $\beta$-glucan \#300 and placebo in children. Fifty children from the Sanatorium for respiratory diseases EDEL were enrolled in the 4-week trial. Twelve children were diagnosed as allergic and asthmatic $(32.5 \%)$ and 28 children suffered from chronic obstructive pulmonary disease (COPD; 67.5\%). The clinical trial was conducted at the Sanatorium EDEL (Zlate Hory, Czech Republic) and the study was approved by the Ethics committees of the Public Health Institute and Sanatorium Edel Czech Republic. This study was performed in agreement with Helsinki declaration (revised version 2000.09.01) and was in full compliance with the rules of for clinical testing for the Czech Republic. Parental consent was given in all cases.

Subjects were randomly assigned to groups which were blinded to intervention. During the intervention period, the subjects consumed $100 \mathrm{mg} / \mathrm{d}$ of $\beta$-glucan or placebo. Both glucan and placebo capsules looked identical. Subjects were routinely evaluated by the medical staff. Ten patients were excluded from the study, due to either an abbreviated stay in the sanatorium or due to a shortened stay based on the parent's request. The observed results are based on 21 children consuming glucan, 19 children consuming placebo.

\subsection{Glucan}

Yeast-derived insoluble glucan \#300 (>85\% dry w/w basis) was purchased from Transfer Point (Columbia, SC, USA). This glucan contains $96 \%$ carbohydrates and $2.1 \%$ proteins. Neutral sugar analysis confirmed $91.3 \%$ glucose and $8 \%$ mannose.

\subsection{Tests}

Children were 8-14 years old with the same average age in both groups. The glucan group consisted of 8 males and 13 females; the placebo group from 7 males and 12 females. In both groups there were an extremely high number of participants exposed to passive smoking (52.4\% in glucan group, $63.2 \%$ in placebo group). All participants absolved at the beginning and at the end 6MWT based on suggested development, including evaluation of additional parameters such frequency before and after physical stress, oxygen saturation, interruption of stress due to the tirednes (Casanova et al., 2011; Karakurt et al., 2010; Lehne et al., 2005; ATSD, 2005). Similarly, evaluation of exhaled nitric oxide was done at the beginning and at the end of the study (Kharitonov et al., 2003; Groneberg-Kloft et al., 2006).

Subjects were observed on a daily basis by the medical staff and the clinical evaluation and the study evaluation were done by the same physician. The safety of the glucan used in our study was done by routine hematologic observations and repeated urine testing. In addition, the regular inspection of oral cavity was performed and evaluated suing the NCI-CTC fading system classification (Lehne et al., 2005).

\subsection{Statistical Analysis}

Statistical significance was evaluated by a pair t-test using a GraphPad Prism 502 software (GraphPad Software, USA).

\section{RESULTS}

Our results are summarized in Table 1. A group of children treated with glucan ( $\mathrm{G}$ group) and with placebo ( $\mathrm{P}$ group) had identical average age, with higher percentage of females in both groups. The majority of participants were diagnosed with COPD $(78.9 \%$ in $G$ group, $61.9 \%$ in $\mathrm{P}$ group). The remainder of the children in both groups were diagnosed with asthma and allergy. A rathersurprising finding was the high percentage of children exposed to passive smoking $(52.4 \%$ in $\mathrm{G}$ group, $63.2 \%$ in $\mathrm{P}$ group), which is app. $30 \%$ higher than at other locations in the Czech Republic at the same age category. This is probably influenced by the socioeconomic background of the families.

The levels of environmental contamination between home conditions and the Sanatorium are significantly different (Fig. 1), with the homes belonging to the most contaminated regions of the country. Data shown in Fig. $\mathbf{1}$ are from the same period and represent an average of three months.

We performed basic clinical evaluation of all children on the first day of the study, including evaluation of physical endurance by 6WMT. The same evaluations were done also on the last day of the study. In G group, we found statistically significant improvements in endurance, whereas the small improvements in $\mathrm{P}$ group were not significant (Fig. 2). 
Vetvicka Vaclav et al. / American Journal of Immunology 9 (3): 88-93, 2013

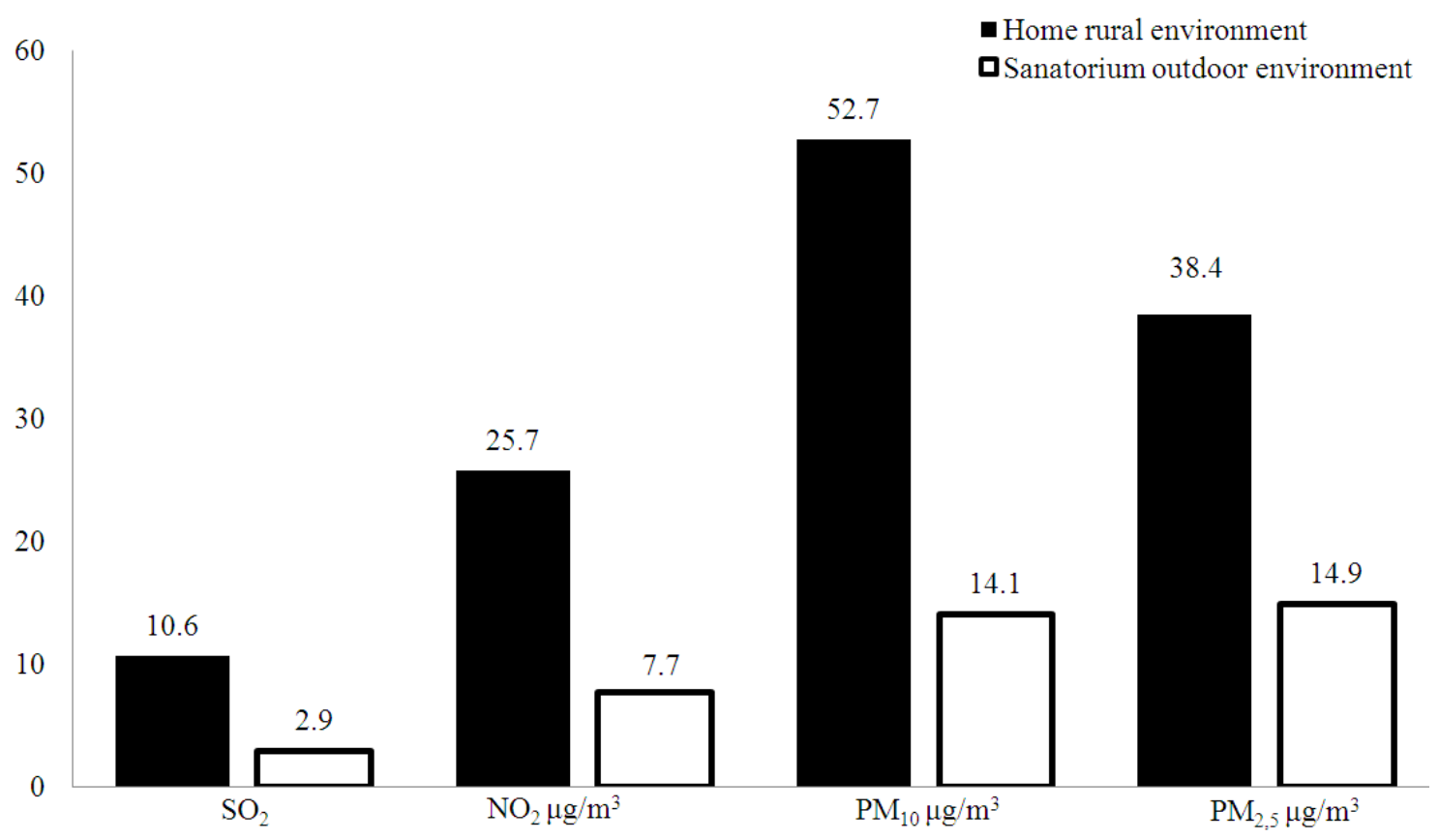

Fig. 1. Meteorological conditions

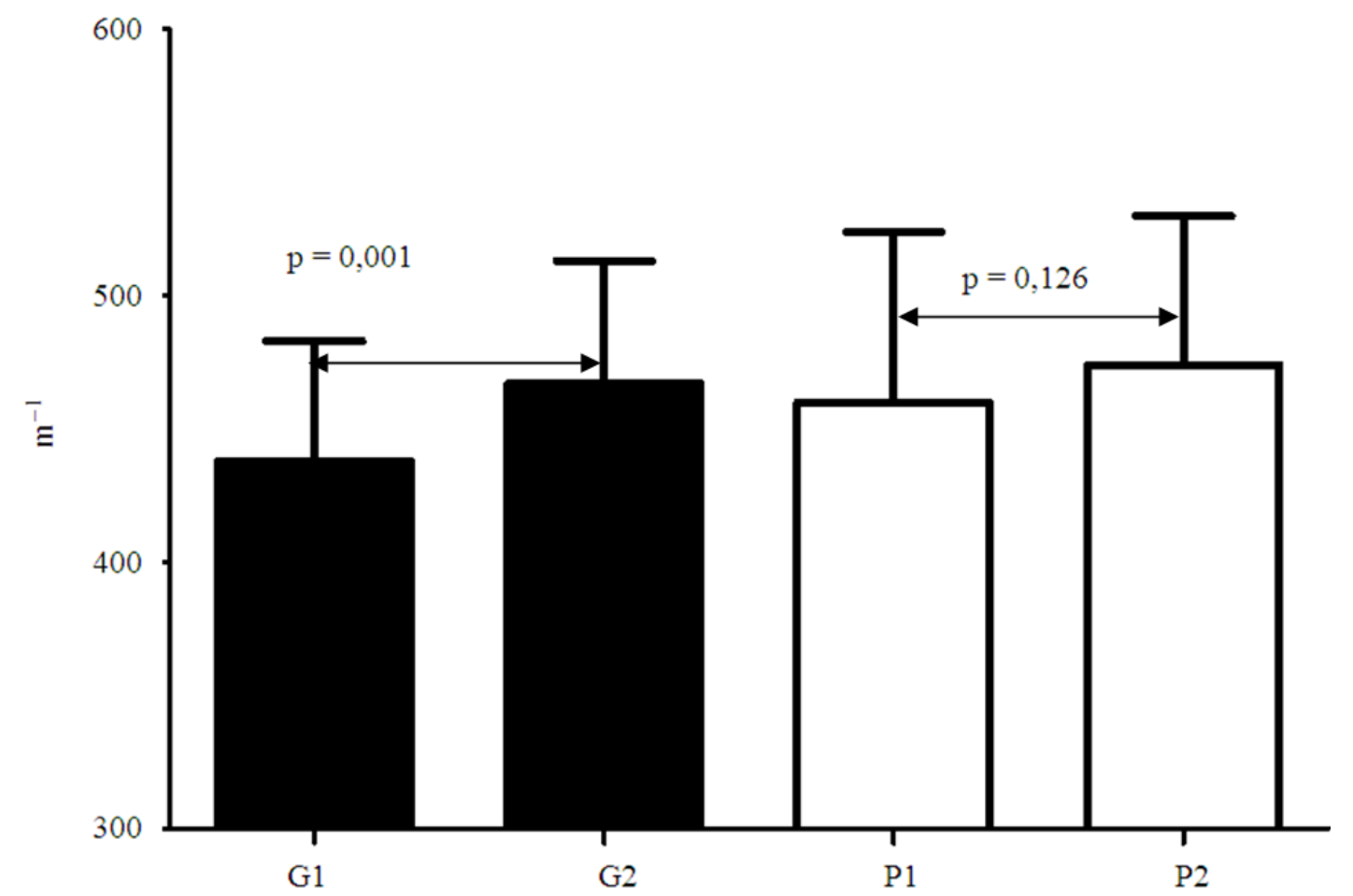

Fig. 2. Six-minute walk test-participants subjects with glucan (G1-day 1, G2-day 30 investigation) and placebo group (P1-day 1, P2 day 30) 
Table 1. Characteristics of the groups of participants (treated glucan and placebo group)

\begin{tabular}{|c|c|c|c|c|c|c|c|c|c|}
\hline & \multicolumn{4}{|c|}{ Glucan group } & \multicolumn{5}{|c|}{ Placebo group } \\
\hline & $(\%)$ & $\mathrm{n}$ & mean & SD & $(\%)$ & $\mathrm{n}$ & mean & SD & t-test \\
\hline Age, year & & 21 & 10,9 & 2,11 & & 19 & 10,68 & 2,29 & 0,754 \\
\hline Gender, male/female & & $8 / 13$ & & & & $7 / 1$ & & & \\
\hline Passive smoking $\%$ & 52,4 & & & & 63,2 & & & & \\
\hline $\begin{array}{l}\text { COPD-Chronic obstructive } \\
\text { pulmonary disease } \%\end{array}$ & 61,9 & & & & 78,9 & & & & \\
\hline Asthma, Allergy \% & 38,1 & & & & 21,1 & & & & \\
\hline BMI-Body Mass Index & & 21 & 20,75 & 3,47 & & 19 & 18,9 & 3,5 & 0,101 \\
\hline 6 MWD, m-day 1 & & 21 & 438 & 45,5 & & 19 & 460,1 & 56,2 & 0,152 \\
\hline Resting heart rate, beats $/ \mathrm{min}^{-1}$ & & 21 & 81,4 & 3,6 & & 19 & 89,7 & 7,6 & 0,001 \\
\hline End of excercise heart rate, beats $/ \mathrm{min}^{-1}$ & & 21 & 92,4 & 2,8 & & 19 & 102,8 & 14,1 & 0,002 \\
\hline Difference in heart rate-beats $/ \mathrm{min}^{-1}$ & & 21 & 12,7 & 10,1 & & 19 & 13,2 & 10,7 & 0,88 \\
\hline 6 MWD, m-day 30 & & 21 & 467,2 & 46,4 & & 19 & 473,7 & 56,2 & 0,069 \\
\hline Resting heart rate, beats $/ \mathrm{min}^{-1}$ & & 21 & 95,5 & 15,1 & & 19 & 97,6 & 13,3 & 0,644 \\
\hline End of excercise heart rate, beats $/ \mathrm{min}^{-1}$ & & 21 & 104,6 & 10,6 & & 19 & 105,4 & 13,4 & 0,834 \\
\hline Difference in heart rate - beats $/ \mathrm{min}^{-1}$ & & 21 & 10 & 12,9 & & 19 & 7,8 & 13,4 & 0,65 \\
\hline eNO 1 (day1) & & 21 & 10,16 & 1,95 & & 19 & 16,07 & 1,88 & 0,0001 \\
\hline eNO 2 (day30) & & 21 & 8,38 & 1,63 & & 19 & 9,02 & 1,6 & 0,212 \\
\hline
\end{tabular}

No significant differences were found in resting pulse frequency (TF), with nonsignificant decrease at the end of the stay in Sanatorium. The levels of exhaled Nitric Oxide (eNO) significantly decreased in both groups. No changes were found during the tests in manifestations of basic diseases, but in the final week of treatment, the findings in $\mathrm{G}$ group were significantly better. At the same time, no negative side effects of either glucan or placebo were observed.

\section{DISCUSSION}

Long-term exposure to environmental pollution, both indoor and outdoor, resulted in the initiation of damage to the respiratory tract with a high risk of asthmatic problems (Groneberg-Kloft et al., 2006). In our experimental groups, we found high exposure, particularly to passive smoking, where the extremely high percentage reflects social and economic conditions of families. Taking the subjects out of the contaminated environment, even for a limited time period, resulted in significant improvements in both clinical and laboratory parameters (Groneberg-Kloft et al., 2006; Rahar et al., 2011; Vetvicka et al., 2013). So far, Renzetti observations of clinical changes in individuals taken out of exposure remains isolated, but compared to our study, used much smaller groups (Renzetti et al., 2009). Our study showed significant improvements of clinical conditions in children supplemented with glucan and agreed with our previous studies showing positive changes in both nonspecific and specific immune responses (Vetvicka et al., 2013). In addition, the environment conditions showed in our study (Fig. 2) demonstrated significantly worse conditions than those in the study (Renzetti et al., 2009). The tested pollen composition did not differ in quality, but we experienced a much higher share of fungal allergens (Renzetti et al., 2009).

No significant differences in age, sex or state of nutrition were found between both tested groups of children. However, we found significant differences in children exposed to passive smoking, therefore we focused particularly on the effects of removing them from levels of exhaled nitric oxide.

At the beginning of the medical stay in sanatorium, the most common diagnosis was COPD, followed by asthma bronchiale. Also quite common was the diagnosis of respiratory allergy, particularly with detected specific IgE antibody response against pollen allergens from trees and grasses.

Initial valuation of lung functions revealed elevated levels of eNO in control (P) group, which was most probably influenced by the exposure to passive smoking. Removing from exposure (both smoking and environmental pollution, most of all exhaust from the traffic) for 4 weeks lead to significantly lower levels of tested functions. To be certain that we eliminated the possibility that the lung functions might be influenced by biorhythm (Kharitonov et al., 2003), we strictly monitored this function at exactly the same time of the day. In 
addition, we correlated the levels of eNO with the activation of inflammatory processes in the mouth, since a strong correlation has been reported (Kharitonov et al., 2003).

A 6MWT test measuring the distance the children covered after 6 min of intensive walking was used for evaluation of physical endurance. Observed levels were significantly lower than found in literature, most probably as a result of chronic respiratory problems (Karakurt et al., 2010; Li et al., 2005; 2007). The physical conditions of children gradually improved during the stay in sanatorium and at the end of their stay, the 6MWT levels were significantly better. This improvement was accompanied by enhancement of immune parameters and general health. These changes are particularly important in young children with lower stress from the exposure to the pollution, both at home and outside. Our finding of 6MWT in the glucansupplemented group were better than in the placebo group and correspond well with the improvements in salivary immunity. As the overall regime of children, including physical challenges, organized rest and nutrition, are identical in both groups, the observed changes were not influenced by these outside parameters (Casanova et al., 2011; Karakurt et al., 2010; Lehne et al., 2005). Therefore, we can conclude that 6MWT test represents a solid tool for measurements of the success of complex spa treatment and glucan supplementation (Lehne et al., 2005; Li et al., 2007).

Our study confirmed the fact that orallyadministered glucans positively influence overall health and regulation of energetic metabolism in children. These effects might be manifested via effects on gut ecology. Due to the rather small groups of children tested in our study, a detailed study with higher recruitment is recommended.

\section{CONCLUSION}

Taken together, our results strongly support the hypothesis that natural immunomodulator glucan significantly improves the health of children with chronic respiratory problems via stimulation of their mucosal immunity. At the same time, no negative effects were observed.

\section{ACKNOWLEDGEMENT}

This study was supported by a Technology Agency of the Czech Republic (\#TA 02020944).

\section{REFERENCES}

ATSD, 2005. ATS/ERS Recommendations for standadized procedures for the online and offline measurement of exhaled lowerer respiratory nitric oxide and nasal nitric oxide. Am. J. Respir Crit Care Med., 171: 912-930. DOI: 10.1164/rccm.200406-710st

Auinger, A., L. Rieda, G. Bothe, R. Busch and J. Gruenwald, 2013. Yeast (1,3)-(1,6)-beta-glucan helpsto maintain the body's defense against pathogens: A double-blind, randomized, placebocontrolled, multicenter study in health subjects. Eur. J. Nutr., DOI: 10.1007/S00394-013-0492-Z

Bergendiova, K., E. Tibenska and J. Majtan, 2011. Pleuran ( $\beta$-glucan from Pleurotus ostreatus) supplementation, cellular immune response and respiratory tract infections in athletes. Eur. J. Applied Physiol., 111: 2033-2040. DOI: 10.1007/s00421-011-1837-z

Casanova, C., B.R. Celli, P. Barria, C. Cote and J.P. de Torres, 2011. The 6-min walk distance in healthy subjects: Reference standards from seven countries. Eur. Respir. J., 37: 150-156. DOI: 10.1183/09031936.00194909

Dennehy, K.M., G. Ferwerda, I. Faro-Trindade, E. Pyz and B. Wevers, 2008. Syk kinase is required for collaborative cytokine production induced through Dectin-1 and Toll-like receptors. Eur. J. Immunol., 38: 500-506. DOI: 10 1002/eji.200737741

Groneberg-Kloft, B., T. Kraus, A. Mark and U. Wagner and A. Fischer, 2006. Analysing the cause of chronic cough: Relation to diesel exhaust, ozone, nitrogen oxides, sulphur oxides and other environmental factors. J. Occup Med. Toxicol., 1: 16. DOI: $10.1186 / 1745-6673-1-1$

Hamano, K., H. Gohra, T. Katoh, Y. Fujimora and N. Zempo, 1999. The preoperative administration of lentinan ameliorated the impairment of natural killer activity after cardiopulmonary bypass. Int. J. Immunopharmacol., 21: 531-540. DOI: 10.1016/S0192-0561(99)00033-8

Jesenak, M., J. Majtan, Z. Rennerova, J. Kyselovic and P. Banovcin, 2013b. Immunomodulatory effect of pleuran ( $\beta$-glucan from Pleurotus ostreatus) in children with recurrent respiratory tract infections. Int. Immunopharmacol., 15: 395-399. DOI: 10.1016/j.intimp.2012.11.020

Jesenak, M., M. Hrubisko, J. Majtan, Z. Rennerova and P. Banovcin, 2013a. Anti-allergic effects of Pleuran ( $\beta$-glucan from Pleurotus ostreatus) in children with recurrent respiratory tract infections. Phytotherapy Res., DOI: 10.1002/ptr.5020 DOI: 10-1002/ptr.5020 
Karakurt, Z., O. Guven, O. Mocin, Y. Kavelioglu and G. Gungor, 2010. Six minute walking distance in kyphoscoliosis patients with chronic respiratory failure. Multidisciplinary Respir Med., 5: 244-249. DOI: $10.1186 / 2049-6958-5-4-244$

Kharitonov, S.A., F. Gonio, C. Kelly, S. Meah and P.J. Barnes, 2003. Reproducibility of exhaled nitric oxide measurements in healthy and asthmatic adults and children. Eur. Respir J., 21: 433-438. DOI: 10.1183/09031936.03.00066903a

Ku, S.K., J.W. Kim, H.R. Cho, K.Y. Kim and S.B. Moon, 2012. Effect of beta-glucan originated from Aureobasidium pullulans on asthma induced by ovalbumin in mouse. Arch. Pharm. Res., 35: 10731081. DOI: 10.1007/s12271-012-0615-8

Lehne, G., B. Haneberg, P. Gaustad, P.W. Johansen and H. Preus, 2005. Oral administration of a new soluble branched $\beta-1,3-\mathrm{D}$-glucan is well tolerated and can lead to increased solivary concentrations of immunoglobulin A in healthy volunteers. Clin. Exp. Immunol., 143: 65-69. DOI: 0.1111/J13652249.2005.02962.x

Li, A.M., J. Yin, C.C. Yu, W. Hung and K.S. Tsang 2005. The six-minute walk test in healthy children: Reliability and validity. Eur. Respir J., 25: $1057-$ 1060.DOI: 10.1164/rccm.200607-883OC

Li, A.M., J. Yin, J.T. Au, H.K. So and T. Tsang 2007. Standard reference for the six-minute-walk test in healthy children aged 7 to 16 years. Am. J. Respir Crit Care Med., 176: 174-180. PMID: 17463419

Novak, M. and V. Vetvicka, 2008. $\beta$-Glucans, history and the present. Immunomodulatory aspects and mechanisms of actions. J. Immunotoxicol., 5: 47-57. DOI: $10.1080 / 15476910802019045$
Rahar, S., G. Sami, N. Nagpal and G.S. Singh, 2011. Preparation, characterization and biological properties of $\beta$-glucans. J. Adv. Pharm. Technol. Res., 2: 94-103. DOI: 10.4103/2231-4040.82953

Ramberg, J.E., E.D. Nelson and R.A. Sinnot, 2010. Immunomodulatory dietary polysacharides: A systematic review of the literature. Nutr. J., 9: 1-22. DOI: 10.1186/1475-2891-9-54

Renzetti, G., G. Silvestre, C. D'Amario, E. Bottini and F. Gloria-Bottini, 2009. Less air pollution leads to rapid reduction of airway inflammation and improved airway function in asthmatic children. Pediatrics, 123: 1051-1059. DOI: 10.1542/peds.2008-1153.

Senoglu, N., M.F. Yuzbasioglu, M. Aral, M. Ezberci and E.B. Kurutas, 2008. Protective effects on Nacetylcysteine andbeta-glucan pretreatment on oxidatice stress in cecal ligation and puncture model od sepsis. J. Invest. Surg., 21: 237-243. DOI: 10.1080/0894.1930802180136

Vetvicka, V. and J. Vetvickova, 2007. Physiological effects of different types of $\beta$-glucan. Biomed. Papers, 151: 225-231. PMID: 18345255

Vetvicka, V. and J. Vetvickova, 2008. A comparison of injected and orally administered $\beta$-glucans. JANA, 11: $42-48$.

Vetvicka, V. and J. Vetvickova, 2013. Reversal of perfluorooctanesulfonate-induced immunotoxicity by aglucan-resveratrol-vitamin $\mathrm{C}$ combination. Oriental Pharm. Exp. Med., 13: 77-84. DOI: 10.1007/S13596-013-0105-7

Vetvicka, V., J. Richter, V. Svozil, L.R. Dobiasova and V. Kral, 2013. Placebo-driven clinical trials of yeast-derived $\beta-(1,3)$ glucan in children with chronic respiratory problems. J. Translat. Med., DOI: 10.3844/ajisp.2013.43.47 